\title{
Using Narrative Cases to Author Interactive Story Content
}

\author{
Ivo Swartjes \\ University of Twente, Human Media Interaction group \\ Enschede, The Netherlands \\ i.m.t.swartjes@ewi.utwente.nl
}

\begin{abstract}
Interactive storytelling is a rapidly emerging field that tries to reconcile story-like experiences with user control. These forces oppose each other, as story implies predetermination and author intent, and interactivity implies freedom and variability. This paper focuses on unscripted (emergent) narrative and addresses the authoring problem resulting from bringing story development into free form interaction. It discusses the possibility of writing story pieces as input knowledge, portraying both believable character behaviour and interesting story situations. It also discusses how such input knowledge can be a source of inspiration for agents in an emergent narrative simulation to improve its potential for story development.
\end{abstract}

\section{Introduction}

The computer forms a new medium to convey stories. Other than in traditional media, such as books and television, computation can be used to bring interactivity into these stories. In this paper, we consider such interactive stories to be stories where a (human) participant can influence the course of the story. Writing an interactive story is a design problem, asking for an investigation of story construction: where can the story offer choices to this participant, and how is the course of the story influenced by these choices?

A traditional way of introducing interactivity to stories is to use branching story lines [11. However, using branching story lines results in an 'explosion of endings': each choice point theoretically introduces a different ending to the story. An added disadvantage is the fact that the whole story is pre-written and therefore has a limited replay value.

The construction of interactive stories beyond the traditional branching story lines calls for more generative descriptions of story content. However, generating an interesting story-like experience is potentially in conflict with generating believable character behaviour [9]. A character must appear consistent and personally motivated for the audience to suspend their disbelief, but personally motivated action does not necessarily help the story forward. The same sort of potential conflict arises when a human participant gains a more central role in the story and can impact its course of events. A user who has freedom of interaction can make choices that conflict with the story intended by the author. This conflict is often referred to as the narrative paradox [1]. 
Ideally, an author of an interactive story should be able to write content that satisfies both sides. An author should be afforded to put his specific ideas about the story into the system, but these ideas should also be represented in a way suitable for use in story-generative processes. This paper proposes an authoring paradigm in which example story pieces are used to influence the event sequence of the story. These pieces are expressed using a semantic representation that enables generative processes to use them. In section 2, some authoring paradigms in the context of interactive storytelling are discussed. In section 3 I propose the use of example story pieces as an alternative paradigm, and discuss the knowledge contained in such story pieces from two perspectives: that of character and that of plot.

\section{Authoring Paradigms in Interactive Storytelling}

Several approaches have been made to address the narrative paradox, each with their own advantages and disadvantages. These approaches can be placed on a spectrum between focusing on autonomous character behaviour on one side, and focusing on engaging plot on the other side.

On one end of the spectrum is a practice to define character behaviour with the aim to make story emerge from it. Two notable projects that follow this approach are the I-Storytelling project 2 and the FearNot! project 1]. The IStorytelling project uses planning formalisms (Hierarchical Task Networks and Heuristic Search Planning) to define character behaviour. The hierarchical definition of tasks and methods roughly corresponds with episodic structures present in simple stories [6] and thus allows for authoring goal-based story content where the real-time interaction of the character's goals allow story variability under the assumption that alternative plans of action are also authored. The focus on character behaviour is even more prevalent in the emergent narrative approach 1 . The FearNot! project is an example, using an affective agent architecture to generate character behaviour portraying bullying scenarios in a primary school setting. The emergent narrative paradigm has no focus on plot whatsoever and the authoring is focused on instantiating the affective models controlling the behaviour of the characters to suit a particular story domain.

At the other end of the spectrum are approaches that use plot requirements to determine the character behaviour necessary to satisfy them. One approach involves constructing a plot using planning operators, and addresses the question how to place character behaviour believably in service of this plot. The authoring focus here is on defining operators that determine a search space for plans in which certain plot goals (e.g., "a bomb goes off") are reached. The (non-interactive) plot planning approach is described in 9]; subsequent effort has shown its applicability in the Interactive Storytelling domain [10]. The preconditions of the planning operators enable the off-line creation of alternative plots for each possible violation of these preconditions by the user.

There seems to be a trade-off between expressiveness and generativity of a particular interactive storytelling system, whereas both seem important. Expressiveness 
is needed to for the system to satisfy the artistic, educational or entertainment goals of the author whereas generativity is important to be able to offer a flexible interactive narrative without the need for hand-authoring many possible plot developments. Highly generative systems require access to the semantics of story content. However, current AI formalisms do not offer the semantics necessary to for instance represent the specific character behaviour of James Bond. Capturing James Bond in a general cognitive architecture seems like a daunting psychoanalytic venture, if at all possible. In designing an interactive storytelling system, a middle ground must therefore be found between generating character behaviour using generic personality models that can respond flexibly to many situations, and writing specific characters with specific behaviours in specific situations. Michael Mateas coins the term Expressive AI to refer to the endeavour of building AI systems that can generate expressive content that conveys the intent of an author [7. The Façade system 8] is a good example of this; its behaviour language allows interactive drama to be authored at beat level. A beat is the smallest unit of dramatic action, i.e., action that helps the story forward. This paper argues for a similar (but more declarative) architectural entity in the form of a narrative case: a semantic representation of an example story piece that portrays believable character behaviour in the context of an interesting story situation. A narrative case aims at representing expressive content with generative semantics.

\section{$3 \quad$ Narrative Inspiration from Example Story Pieces}

The practice of using examples to capture and reuse knowledge in a certain domain, rather than a full specification of the domain knowledge, is referred to as Case Based Reasoning (CBR). Up till now, CBR is used only sporadically in interactive storytelling or story generation projects. The interactive storytelling system OPIATE uses CBR to achieve morphological plot variation [3, similar to [4. MINSTREL [14] is model of story generation using CBR to modify and recombine predefined episodic knowledge structures to construct stories. Its story generation process is guided by author goals, such as illustrating an intended theme, or fixing consistency problems in a story under construction.

Now, the core value of CBR is that knowledge is captured in terms of examples (rather than extensive domain knowledge). From the standpoint of Expressive AI, it offers authorial affordances, in that these examples can express the intentions of the author, and can as such be used as inspiration by a system that tries to deliver a story-like experience. If example story pieces are formulated in terms of elements that can also be generated 11 and as such be incorporated in the emergent unfolding of a story, we allow a framework that has the flexibility to produce narrative on the spot to be infused with authorial intent, a process I would like to call narrative inspiration [13].

When the narrative cases (expressing example story pieces) are authored with two principles in mind, namely (1) that they express believable behaviour, and

\footnotetext{
${ }^{1}$ When using affective architectures as in emergent narrative, these are elements like emotions, goals, actions, events and beliefs.
} 
(2) that they portray interesting specific situations as desired by the author, a collection of such cases can be used as information source for interactive storytelling applications. To this end, my aim is to integrate the use of narrative cases with the emergent narrative approach of the Virtual Storyteller [12. The Virtual Storyteller simulates affective character agents and uses a plot agent to manage the unfolding narrative, trying to steer it in a promising direction. The semantics of our narrative case representation is based on causality between story elements [13. The unfolding narrative is also represented in terms of causality. Rather than using the narrative cases as (partial) solutions to author goals (as done in MINSTREL), I want to integrate the causal knowledge that these cases contain with the processes that the agents in the Virtual Storyteller already run. I will briefly discuss such processes from both perspectives (from a generative point of view), and show how these processes can be informed by narrative cases. When using narrative cases as knowledge sources, I hope to end up with narrative that is still believable and more interesting than can be achieved without the use of these cases.

\subsection{The Character Perspective}

In the Virtual Storyteller, we follow the work that has been done by Bryan Loyall on designing believable characters [5. Some of the requirements for believable characters are that they express personality and emotions, appear self-motivated, change by experience, form social relationships, are consistent and portray the 'illusion of life'. I will address the causal aspects of two of these believability requirements: self-motivation and emotion.

It is important that character actions appear motivated by their own personal goals or emotions [9]. When a character tries to achieve goals, the question is what actions or subgoals it should choose to achieve them. The aim of 'standard' AI task planning algorithms (intended for effective and efficient achievement of intentions) does not necessarily match the aim of dramatic task planning, where ways to achieve goals are determined by a dramatic perspective. For instance, James Bond might have a very specific way to dismantle a villainous organization, a high-level goal that is not only awkward to express in primitive planning operators, but is also typically something an author might want to have specific control over. Narrative cases can express how these high-level goals might decompose; the lower-level physical details lend themselves better for planning approaches using means-end reasoning, since they arguably exist mainly to afford the dramatic action.

The second process that contributes to character believability is that of emotional reactions to events. Within the affective agent architecture of the Virtual Storyteller, a character first adopts beliefs based on perceptions which will then result in emotional states that affect how it proceeds to act. Such processes are currently based on realism, but can also be informed by what the story needs. For instance, in Shakespeare's "Romeo and Juliet", when Juliet sees Romeo faking his death, her belief in his death (a believable reaction) has dramatic consequences (i.e., she commits suicide). A more realistic but maybe less interesting response might have her check his pulse. By modelling the relationship between 
perceptions, beliefs and emotional reactions from an author perspective, a character's internal world can be enriched by a case base of dramatically fertile experiences. This also makes it possible for characters to make quick assumptions about other characters' internal worlds. They can make plans to affect each other's beliefs without complicated adversarial reasoning.

A narrative case effectively defines specific character behaviour within a certain narrative context that makes it believable and helps the story forward. If this context is met, a character can respond according to the case rather than according to the result of its own deliberative processes, thus contributing to the emerging story.

\subsection{The Plot Perspective}

The plot agent is responsible for helping the story forward where necessary. Within the emergent narrative simulation, the unfolding narrative can be influenced without breaking the autonomy of the characters, when environmental control is used. Two ways of environmental control that can be inspired by narrative cases, are the following: making events occur that (possibly) affect the plans of characters, and real-time construction of the story world.

The first way of environmental control raises the question which events will contribute to an interesting plot. These are for instance events that thwart or help a character's goals and plans, or cause an emotional reaction. Narrative cases can express how character action results in events (e.g., James Bond cuts himself when he breaks through a window), and how these events can cause interesting or desired character reactions. Both might form a basis for the drama manager to select and introduce them in the story.

The second way of environmental control involves filling in the details of the story world setting, which does not need to be fully fixed from the start. Theoretically, only information conveyed to a user at a certain point in time constrains the possible setting the story world can be in. This allows the introduction of characters, objects and such when needed for plot development (this idea has been explored in a story planning domain [9]). The drama manager can use the narrative cases as inspiration to decide what knowledge to introduce at a certain point in time: certain cases will become applicable for the unfolding of the story (e.g., introducing a window in the house of the villain when James Bond arrives makes it possible for him to break in). The idea here is that the setting exists in service of the plot, rather than as a cause of it.

On the assumption that a narrative case expresses a course of events as the author intends it, trying to achieve this course of events is a high priority for the plot agent. The plot agent keeps track of the unfolding story and can opportunistically detect a context in which these cases can apply, or try to achieve such contexts more actively.

\section{Conclusion}

This paper puts forward the idea of having an author express his intent for the unfolding of an emergent narrative by combining representations of believable 
character behaviour with interesting narrative situations, resulting in a knowledge structure called the narrative case. Authoring such cases is less awkward than specifying high level domain knowledge, and integrating them with an emergent narrative architecture increases the potential of the author's intent being reflected in the actual simulation. A more detailed description of the use of narrative cases for the Virtual Storyteller is given in 13] subsequent research will focus on actual experiments to investigate the effects of offering narrative inspiration to the processes that the agents of the Virtual Storyteller already run.

\section{References}

1. Aylett, R., Louchart, S., Dias, J., Paiva, A., Vala, M., Woods, S., Hall, L.: Unscripted narrative for affectively driven characters. IEEE Computer Graphics and Applications 26(4), 42-52 (2006)

2. Cavazza, M., Charles, F., Mead, S.J.: Character-based interactive storytelling. IEEE Intelligent Systems 17(4), 17-24 (2002)

3. Fairclough, P., Cunningham, C.R.: AI structuralist storytelling in computer games. Technical report, University of Dublin, Computer Science Department (2004)

4. Gervás, P., Díaz-Agudo, B., Peinado, F., Hervás, R.: Story plot generation based on CBR. Knowledge-Based Systems 18(4-5), 235-242 (2004)

5. Loyall, A.B.: Believable Agents: Building Interactive Personalities. PhD thesis, Carnegie Mellon University, Pittsburgh, PA (1997)

6. Mandler, J.M., Johnson, N.S.: Remembrance of things parsed: Story structure and recall. Cognitive Psychology 9, 111-151 (1977)

7. Mateas, M.: Expressive AI. Leonardo: Journal of the International Society for Arts, Sciences, and Technology 34(2), 147-153 (2001)

8. Mateas, M., Stern, A.: Façade: An experiment in building a fully-realized interactive drama. Technical report, Literature, Communication and Culture and College of Computing, Georgia Tech. (2003)

9. Riedl, M.O.: Narrative Generation: Balancing Plot and Character. PhD thesis, North Carolina State University (2004)

10. Riedl, M.O., Stern, A.: Believable Agents and Intelligent Story Adaptation for Interactive Storytelling. In: Technologies for Interactive Digital Storytelling and Entertainment (TIDSE) (2006)

11. Riedl, M.O., Young, R.M.: From linear story generation to branching story graphs. IEEE Computer Graphics and Applications 26(3), 23-31 (2006)

12. Swartjes, I., Theune, M.: A Fabula Model for Emergent Narrative. In: Technologies for Interactive Digital Storytelling and Entertainment (TIDSE) (2006)

13. Swartjes, I., Vromen, J., Bloom, N.: Narrative inspiration: Using case based problem solving to support emergent story generation. In: Proceedings International Joint Workshop on Computational Creativity (2007)

14. Turner, S.R.: The creative process: a computer model of storytelling. Lawrence Erlbaum Associates, Hillsdale, NJ (1994) 